\title{
An Efficient Data Science Technique for loT Assisted Healthcare Monitoring System using Cloud Computing
}

\section{Karthikeyan}

Valliammai Engineering College

Samydurai Arumugam ( $\nabla$ samyduraia.cse@valliammai.co.in )

Valliammai Engineering College

\section{Research Article}

Keywords: loT, cloud computing, data science, improved pigeon optimization, feature selection, healthcare monitoring

Posted Date: July 12th, 2021

DOI: https://doi.org/10.21203/rs.3.rs-644765/v1

License: (c) (i) This work is licensed under a Creative Commons Attribution 4.0 International License.

Read Full License 


\title{
An efficient data science technique for IoT assisted healthcare monitoring system using cloud computing
}

\author{
L. Karthikeyan ${ }^{1 *}$, and A. Samydurai ${ }^{2}$ \\ ${ }^{1}$ Department of Computer Science and Engineering, SRM Valliammai Engineering College \\ SRM Nagar, Kattankulathur- 603 203, Chennai, Tamil Nadu, India \\ [karthikeyanl.cse@valliammai.co.in] \\ ${ }^{2}$ Department of Computer Science and Engineering, SRM Valliammai Engineering College \\ SRM Nagar, Kattankulathur- 603 203, Chennai , Tamil Nadu, India \\ [samyduraia.cse@valliammai.co.in \\ *Corresponding author: L.Karthikeyan
}

\begin{abstract}
In this era of smart healthcare system, patient expects better healthcare support with low cost which satisfy through the innovative process such as internet of things (IoT), cloud computing and data science techniques. Meantime, the healthcare industry faces many problems including the data collection and storage for further progress. For healthcare monitoring system, the data collection and data analytics plays important role to screening the patient health. Therefore, data science techniques and cloud computing are heart for the healthcare system to resists several problems in terms of technical aspects. For further enhancement, efficient data science technique is proposed for IoT assisted healthcare monitoring (DSTHM) system using cloud computing, which improves the data processing efficiency, data accessibility in cloud. The several IoT sensors are used in a personcorpseto collect the real clinical data. The composed data are then maintained in cloud for added data science processing. In DST-HM system, we first introduce a modified data science technique i.e. improved pigeon optimization (IPO) algorithm for grouping the cloud stored data which enhances the prediction rate. Second, we illustrate the optimal feature selection technique for feature extraction and selection. Then, a backtracking search based deep neural network (BS-DNN) is used to classify the healthcare of human. Finally, the performance of proposed system is analyzed through different real-time healthcare dataset and the consequences are comparing with the existing state-of-art healthcare monitoring systems.
\end{abstract}

Keywords IoT, cloud computing, data science, improved pigeon optimization, feature selection, healthcare monitoring

\section{Introduction}

The next wave of the computing age is transcending the boundaries of the conventional desktop. In the Internet of Things (IoT) model, numerous of the substances around us are in the network in some form. In response to this new challenge, Radio Frequency Identification (RFID) [1], and touch network technologies are evolving, in which in sequence and announcement systems are imperceptiblyentrenched in the surroundingsapproximately us. These consequences generate the data needed for storage and distribution in a collaborative, efficient, and understandable format. This model will have the same products and services offered by traditional products [2].Knowledge of Intelligent Network and Computer Environment with network capital is an important fraction of IoT. By the time the couple created 4G-LTE wireless internet, the development of global information and communiqué networks was previouslyobvious [3]. though, for visual success, it is essential to develop a computational model beyond traditional mobile computer monitors that can be used to integrate everyday objects with smart phones and small systems and to integrate intelligence into our environment [4].Proper medical care under patient 
supervision is considered important to improve quality of life and ensure effective health and community care [5]. Extensive applications and services such as mobile telemedicine, patient monitoring, medical care, emergency response and management, and comprehensive monitoring of personal monitoring and health information are of great benefit to patients and medical staff [6-7].

Research has shown that cloud computing is an efficient and economical technology that enable largescale data incorporation and investigation for genetic tablets research [8]. Considering the process of collecting essential patient data, Rolim et al entail more effort to assemble, input and analyze in sequence suggests a cloud-based system for process automation. The American Professional Network (AON) [9 11], improves patient care digitally using health records and updates clinical procedures using the cloud app. Although many successful cloud computing projects have been implemented in the health sector, there are several obstacles to its complete control. In this study, the author discuss the challenge of cloud computing and suggests a cloud computing strategic planning model that can be used by an association to decide cloud project course, approach, and resource allocation [12].The Internet of Things (IoT) [13] has elegant nodes that can understand and gather information in genuinepoint in time for tracking. It is an important system of remote health monitoring that poses a great threat to the security and performance of clinical data [14] [15]. In addition, IoT devices control caching, computing, and sensitivity resources, so protected and proficientcheckup data recovery is the solution to the reserve control quandary [16]. Compare to IoTcampaign, Edge Cloud Edge campaign have a lot of caching and dominanthanding outcapability, so Edge confusemust be the best result for overcoming the limitations of IoT device storage and system resources. Additionally, the cluster can improve the sensitivity capability of IoTplans by distribution the capital of cluster memberand their collaborations [17]. IoT manages data distribution using IP-based endo-end approaches, in which each user performs a free data communiquéprocedure to recover data from aexactmachine defined at the target speak to [18]. This lead to an effective recovery of checkup data for the subsequentreason [19] Users must perform data redistribution processes to obtain medical data, resulting in data overload and data recovery. The target device is optimal or the consumerhave to obtain checkup data from the aimmachine. This further aggravates the inability of data recovery [20].

The main intension of this article is to develop an IoT assisted healthcare monitoring system using cloud computing that enhances the data processing efficiency and data accessibility in cloud. The further sections of this article are arranged in the follow setup. Section 2 represents the thorough review of writing regarding the IoT healthcare monitoring systems. The proposed data science technique for IoT assisted healthcare monitoring (DST-HM) is described in segment 3. The presentation of the planned system is evaluate in section 4 and the conclusion of the article is represented in section 5.

\section{Related work}

Apoorva et al [21] have proposed the Cohort Intelligence (CI) method is the best approach to improving the design problems of controlled engineering. Instead of the usual repair methods, it uses a probabilitybased synthetic control approach that reveals the inherent rigidity of CI technology. The approach is confirmed by solving three design problems. The results of this review can be compared with simulation analysis based on particle mass optimization (SiC-PSO) and particle co-evolution optimization (CPSOSA) (Cagnina et al., Informatica.

Saha et al [22] have proposed a network technology and the Internet of Things (IoT) have made human life easier. In addition to a "smart" environment that includes smart homes, smart cities, and smart farms, IoT has added health systems, direct diagnosis, and medical advice. Fog layers are used to improve the capability of IoT-based healthcare system, and they demonstrate its importance with rapid response times and short delays. However, such incidents are to some extent related to security / privacy issues for user privacy. Such technology has been operating within the framework of privacy since childhood. Therefore, 
our current job is to create Electronic Medical Records (EMRs) within an e-health framework that protects privacy. In addition, we explored specific task response times and time delays compared to recent tasks.

Suneeta et al [23] have plannedProvides a structure for the use of IoT and ALERT in the health sector. The aim of the study is to create a low-cost steadfastexample that monitoressentialparameter of the human body, transmits data to mania speak or Cloud, and notifies the patient through the appropriate application. The aim of this study is to recover the patient's familiarity in understanding health status. The new health care system in the biological body represents a shining place in the medical field as it shapes the relationship between doctor and patient by analyzing the relationship between the biological conditions in the body.This method help the physician to correctly diagnose the disease and provide emergency services to the health system. This framework facilitates the use, application and quality of real-world technology. The advanced ALERT scheme helps to take preventive events; this allows the client to receive emergency medical care.

Haghi et al [24] have proposed Introduced IoT based platform that is flexible, widely used, durable and user-friendly in terms of environment, physiology and behavior. In adding, we introduce an inventive wrist prototype wrist watch for environmental monitoring. IoT-Gateway has been developed as an intermediate hub between data and collection, enabling efficient and final communication for data collection and synchronization between users and physicians. On the one hand, smartphone physicians such as IoT-Gateway define tasks, operate on devices, select calibration settings, and determine the length of on / off sensors; Distributors are not limited to individual users.

Wu et al [25] have proposed a tiny sensor is designed to measure a variety of physiological signal, such as an electrocardiogram (ECG), photoplasmograph (PPG), and body hotness. Because electrocardiography and PPG sensors are included into a single machine, a special feeler patch can be worn to measure pulse arrival time (PAT) and blood pressure without the need for additional lines and equipment. The sensor patch contains three sensors for central signal acquisition, a processing panel and a complex monitor of the signal for the supply and distribution components. All components are designed in a rigid framework, making it easy for the human body to interact with remote health monitoring programs. Consumption A specific physiological signal to reduce energy consumption

Kumar et al [26] have proposed Explore optimization algorithms that are compatible with Healthcare 4.0 trends, and improve blockchain-based decentralized application for the stylish healthcare scheme. In addition, smart contract are designed to build trust in their specific system and speed up payment methods. This work involves the simulation and implementation of a modified draw near. Imitationfallout show that the necessary gas worth (indicating volume and value) is within the present range of the ether network gas. The specified system is active because the volume usage is over $80 \%$. The automatic execution of the contract takes less than 20 second. A good digit (standard of 3 simulation times) is generate in a network that represents fitnessantagonism. Even though simulation and performance errors ranged from $0.55 \%$ to $4.24 \%$, these errors did not affect the overall presentation of the system due to variations in simulation and lack of actual (complex) data.

Wang et al [27] have presented a protected health monitor framework that integrates NDN-based IOT and cloud computing. The NDN program uses this framework to get better the efficiency of medical data recovery, and uses cyber text and signatures to make sure the safety of medical data communication. The structure is evaluated as a criterion. The data show that the delay and cost of medical data recovery were reduced by $28 \%$ and $52 \%$, respectively, compared to the current data.

Randa et al [28] have proposed a MobMBAR is the traffic distribution planning and distribution approach in IoT Healthcare Architecture with cloud-fog computing models. MobMBAR provides lively cleaning 
functions between cloud and fog strategy. It is a data location based draw near based on the conversion of estimated site data into real life. It takes into explanation the preferences of the tasks specified in the classification (class) and the greatestretort time for the maximum response. Therefore, it has a high planning priority until it has the highest categorization and the lowest response to the task. The specific approach allows QoS to ensure reliable processing time of many medical services, including specific processing speeds, data sizes, and numbers.

Anuradha et al [29] have presented this feature focuses on improving and implementing health calculations. It creates a framework for civilizing the presentation of the healthcare sector around the world. By keeping all medical data in the cloud, the limitations of traditional medicine can be defeat. Encryption and encryption are performed using the AES algorithm to identify and protect the treatment of cancer patients. The foremost focus is on effectively managing health data when the patient is not at home, because the information needed for cancer treatment is stored in the cloud. The use of VMs significantly reduces the time to 400 to 160 work completion. Cloud SIM provides an adaptive simulation framework that enhances visual and background effects.

Mahbub et al [30] have proposed integratedillumination and airing system using HTTP protocol based on smartphones or computers. Additionally, users can monitor the cloud from anywhere in the world by accessing data directly from a computer server. As it is a wireless gadget, it communicates with the management system via GSM and Wi-Fi network using modern HDTP protocol. In addition, these works show the computer's recommended consumption, consumption power consumption, economic analysis, and its performance. The article also provides an overview of early IoT infrastructure.

\section{Problem methodology and System architecture}

\subsection{Problem methodology}

Anuradha et al. [31] containFocuses on improving and implementing health calculations. It offers a framework used to improve the performance of the healthcare sector around the world. Traditional medicine is out of bounds. The major goal of this study is to use the IoT system in the cloud medical meadow, such as the electronic health scheme, to monitor patient details. The patient's name, age, speak to, wedded status, telephone digit, preceding olden times, blood mass and heart speed abnormalities are composed by various sensory plans used in the patient's body. For safety and evaluation, it was decided to encrypt the blood test consequences of growth patients and store them in the obscure archive. Encryption and encryption are performed using the AES algorithm to identify and protect the treatment of cancer patients. The main focus is on effectively managing health data when the patient is not at home, because the information needed to treat cancer is stored in the cloud. In this age of healthy health, the patient expects better health care at a lower cost and is satisfied with innovative processes such as the Internet (IoT), cloud cloud computing and data science technology. At the same time, the health sector faces a number of problems, including data processing and storage for further development. For the health monitoring system, data collection and data study play an imperative role in the health screening of patients. Therefore, data science tools and cloud computing are technically addressing many of the problems in the health center. For further improvement, effective data science technology is recommended for the IoT Assisted Health Monitor (GST-HM) system with cloud computing, which improves data processing performance and improves data access to the cloud. IoT uses multiple sensors to collect real medical data from the human body. The composed data is then store in the cloud for data processing. The main donations of planned DST-HM system summarize as follows:

- For the first time on the DST-HM system, we are introducing modified Data Science Techniques or improved pigeon optimization (IPO) algorithm to compile data stored in the cloud, which improves forecast speeds. 
- Second, we describe the optimal features for obtaining and selecting characteristics.

- A Deep Neural Network (BS-DNN) based on backlink search is used to classify human health services.

- Lastly, the performance of the proposed system is analyzed in real time in various health databases and the results are compared with modern health monitoring systems.

\subsection{System architecture of proposed DST-HM system}

Fig. 1 Computer architecture provides useful data science data technology with the help of IoT for Health Monitoring System (DST-HM). Various sensors are used to monitor changes in human body hotness and blood heaviness. The serene data will be store using any local dispensation method that is considered to be genuine medical data. This particular system is very effective in predict cancer, so it can be used to make a blood test result or mammogram after detecting blood cell and temperature changes. Since the characteristics of the blood test are the characteristics of a normal person or a person with cancer, the characteristics of the blood test results are shown for classification. This is necessary in view of the fact that certain benefits may accrue to both users and certain users or certain types of users.

The source determines how users can use remote sources. Computers are delivered through client-planned contracts such as workstations, computers, tablets, and cell phone. A small portion of these tools - cloud customers - all depend on a distributed computing system or most of their software. Instead of using a web browser to integrate with the cloud application, do not bother with the client's explicit programming cloud application. It is a good idea to use in-depth learning tools such as Alexnet, VGG16 and Googlenet for classification. By designing the structure of a limited neural network, it is possible to identify different types of tumors, such as breast cancer, lung cancer, and leukemia. If the data is store in the obscure, the enduring does not require to constantly requesting treatment details from the hospital. It has direct access to health data store in the cloud at any time without interruption or major processing. 


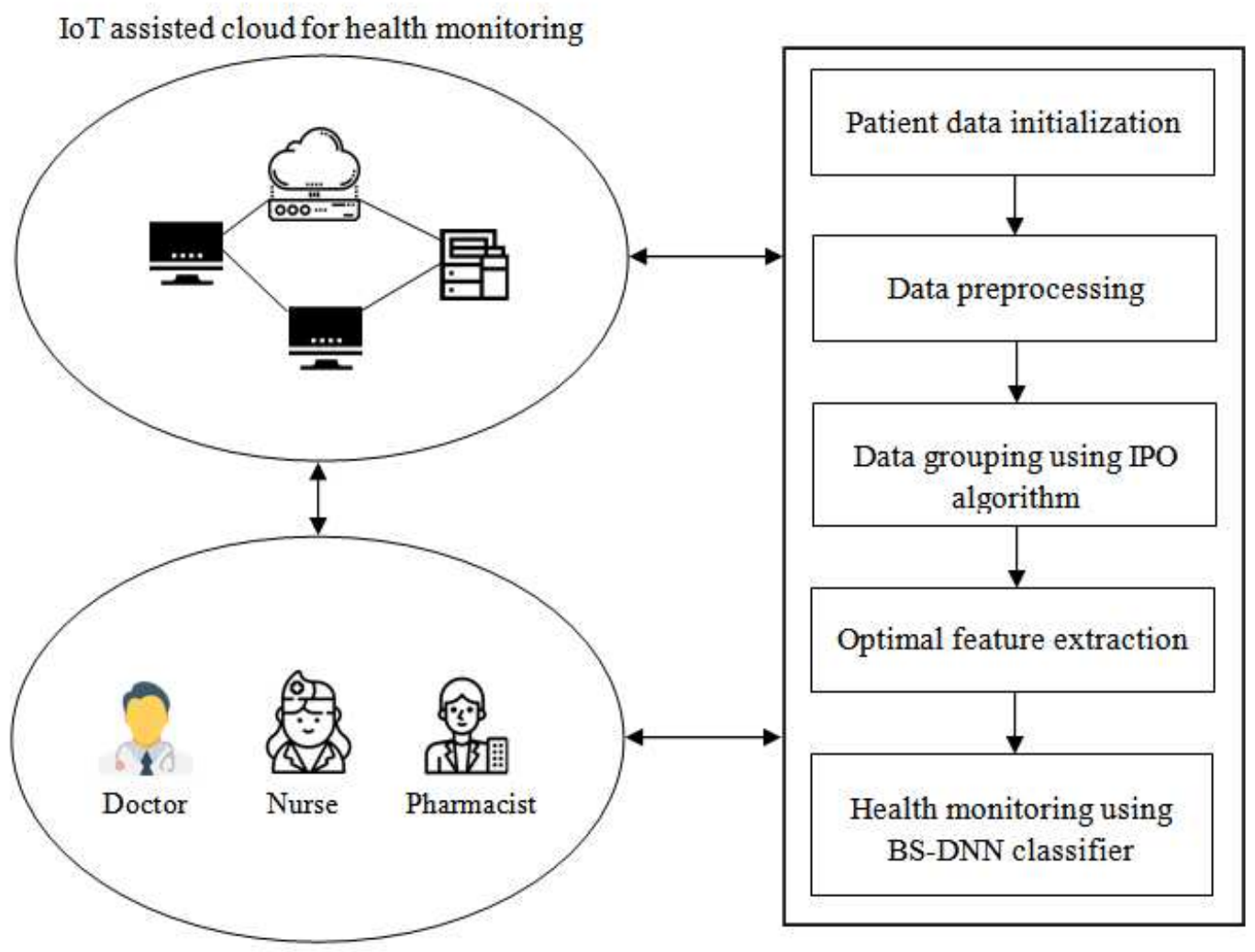

Fig. 1 working function of planned DST-HM system

\section{Proposed DST-HM technique}

\subsection{Grouping the cloud stored data using improved pigeon optimization (IPO)}

PIO algorithm the newly suggested SI algorithm reflects the pigeon's incoming features [32].In particular, two operators were developed using precise rules for the incoming properties of a pigeon: (1) a map and scopeworker reflecting the incoming behavior of the sun and magnetic particles; (2) As a stage operator, the incoming behavior takes place in a familiar place. The action of a magnetic field allows you to determine the direction, height, or position of a magnetic field. Pigeons can understand the earth field using a magnet to create a brain map. In their view, the compass to change direction is the height of the sun. They rely heavily on magnets and the sun as they are in untried places far away from house. The map and scopeworker are intended to reflect this incoming behavior. Particle swarm optimization (PSO) is a classic effective meta-chorister training method. enthused by the Hybrid Dragonfly Algorithm, we offer the PSO algorithm and the new IPO learning algorithm to enhance optimization capabilities and global search capabilities. The process for learning the IPO algorithm is described as follows;

Launch PSO settings, update PSO settings, and enable adaptive mutation functionality in PSO. The pieces in the PSO start from the specific probability of each contact. Adaptive mutation functionality can enhance PSO demographic diversity and expand particle search space. The formula for updating PSO speed and status is as follows:

$$
\begin{gathered}
P_{m}(T+1)=\alpha \cdot P_{m}(T)+a_{1} \cdot \operatorname{rand}_{1} \cdot\left(V_{i}(T)-Q_{m}(T)\right)+a_{2} \cdot \operatorname{rand}_{2} \cdot\left(V_{s l}(T)-Q_{m}(T)\right) \\
Q_{m}(T+1)=Q_{m}(T)+P_{m}(T)
\end{gathered}
$$


Here $P_{m}(T)$ and $Q_{m}(T)$ signify the speed and location of the $m^{\text {th }}$ atom in the $T^{\text {th }}$ iteration. $\alpha$ is the inertia weight, $a_{1}$ and $a_{2}$ characterizespeeding upfactor in PSO, and the values of $a_{1}$ and $a_{2}$ are constantly greater than $0 . V_{i}$ is the character best location and $V_{s l}$ represent the global best place. rand and rand $_{2}$ are two random figuresamong 0 and 1 .

Initialize the parameter of IPO algorithm and include their worldwidebest morals. If the global best principles of the PSO are improved than the actual principles of the IPO, then the IPO algorithm achieves the best global values of the PSO.

IPO is used to update settings. The change weight is included in the IPO algorithm. The weight of the recession will affect the balance between global and IPO local searches. The change weight formula is defined as follows:

$$
\alpha(T)=\alpha_{u}-\left(\alpha_{u}-\alpha_{z}\right) \times T / t
$$

Here $\alpha(T)$ is the inactivityheaviness in the $T^{\text {th }}$ iteration. $\alpha_{u}$ Represent the initial inactivity weight and $\alpha_{z}$ is the inertia heaviness when the iteration stops. $t$ Represents the utmost number of iterations.

The IPO algorithm consists of two different operator - a map, a scope operator, and a landmark operative. The formula for updating speed and position on the map and rangeworker is shown below as:

$$
\begin{gathered}
P_{n}(T+1)=\alpha(T) \cdot P_{n}(T) \cdot z^{-R T}+\operatorname{rand}_{3}\left(Q_{s}-Q_{n}(T)\right) \\
Q_{n}(T+1)=\left(Q_{n}\right) T+P_{n}(T+1)
\end{gathered}
$$

Here $P_{n}(T)$ and $Q_{n}(T)$ correspond to the speed and location of the $n^{\text {th }}$ pigeon in the $T^{\text {th }}$ iteration. rand isaaccidental number among 0 and $1 . R$ is the map and scope factor, whose charge is among 0 and 1 .

When the map and extentoperativereach the greatest number $t_{1}$, the markeroperative starts to find the finestanswer. The updatemethod of pigeon'splace in the landmark operative is described as follows:

$$
\begin{gathered}
J_{\text {LANDMARK }}(T+1)=\frac{J_{\text {LANDMARK }}(T)}{2} \\
Q_{p z j}(T)=\frac{\sum Q_{n}(T) \cdot s\left(Q_{n}(T)\right)}{J \cdot \sum s\left(Q_{n}(T)\right)} \\
Q_{n}(T+1)=Q_{n}(T)+\operatorname{rand}_{4} \cdot\left(Q_{p z j}(T+1)-Q_{n}(T)\right)
\end{gathered}
$$

In the procedure of the landmark worker, the quantities of pigeons decrease by half in each iteration. $J_{\text {LANDMARK }}(T)$ represent the number of pigeons in the $T^{\text {th }}$ iteration. $Q_{p z j}$ is the center position of the pigeons and $s\left(\right.$.) represent the fitness purpose. $r a n d_{4}$ is a random number between 0 and 1 .

When the IPO algorithm reaches the maximum landmark operator $\left(t_{2}\right)$, the IPO algorithm terminates and the end result of the optimization of the global optimal solution $\left(Q_{s l}\right)$ location parameter. 
In our model, the Gaussian breach was extra to the recently created pigeons, which increases the likelihood of local optimization. In addition, when repeating, the Gaussian component should be used with a high probability of low probability. Thus, individuals were added to the Gaussian barrier as:

$$
Q_{m s}(T)=Q_{m}(T)+\log S I G\left(\frac{J p_{\max } / 2-T}{y}\right) J(\beta, \gamma)
$$

Where $\log \operatorname{SIG}($ ) denotes a logarithmic sigmoid transfer meaning, which decrease the amplitude of the Gaussian commotion as the iteration profits.

In the replica of the simulated annealing, the poorerpersonality is kept back with the likelihood $X_{c}$. In this study, assume that the dissimilarityamong the condition of the personage added Gaussian disturbance $Q_{m s}$ and the old one $Q_{m}$ is $\Delta f$, and the EPF based similar is the maxim optimization quandary, then the probability $X_{c}$ is define as

$$
X_{c}=\exp (\Delta k / t)
$$

Where tis an annealing heat, it decreases with increasing rearrangement. An innovative algorithm allows you to exit the local optimal if the firsthotness is high and the annual is low.

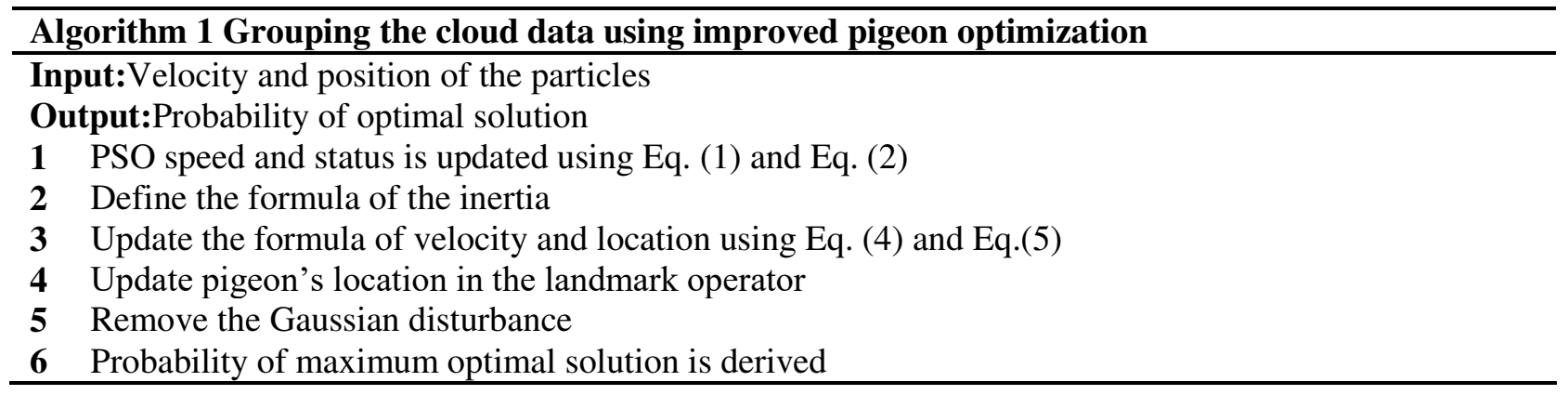

\subsection{Feature extraction and selection}

Characteristic Extraction is the selection of an input picture in a miniature format that retains the features of the original image. Once identified, it is considered to have reduced the acquisition of characters. The most active input properties can be classified as "attribute extraction" and "attribute selection", which reduce class variance and increase class variables. Another definition of attribute extraction is "the process of obtaining obvious inverse attributes from digital codes". This eliminates the most important data for taxonomic purposes, reduces class structure variability and increases class structure variability. What sets it apart from other characters is the search for input properties and features for each character.

PCAs are often used to present small amounts of information at a small loss. Smaller space will reduce storage usage and calculation costs [33]. Known a multivariate time series, they first change it into a Univariate time series: 


$$
\hat{P} \in B^{x}, \quad x=R \times F
$$

It can be unspoken that each input window section has $x$ scope. After that, a $x$-dimensional connote vector can be represented as

$$
\alpha \in B^{x}
$$

The mean vector $\alpha \in B^{x}$ now is calculated as:

$$
\alpha_{c}=\frac{1}{Q} \sum_{e=0}^{Q-1} d_{c}^{e}, c \in[0, x-1]
$$

$Q$ is the number of sample used to train the PCA. After that, the covariance matrix of the whole preparation dataset is intended as

$$
\sum=\frac{1}{Q-1}\left((D-q)^{t}(D-q)\right)
$$

Eigenvectors $\left(E_{1}, E_{2}, \mathrm{~K} E_{D}\right)$ and corresponding Eigenvalues $\left(\lambda_{1}, \lambda_{2}, \mathrm{~K} \lambda_{x}\right)$ that gratify equation are compute.

We choose $K<x$ eigenvectors with the biggest eigenvalues to build a $x \times K$ matrix $J$. The measurement of all new samples $d$ can be abridged by apply this formula:

$$
q=J^{t} \times d
$$

To the end, every input multivariate time sequence will be represent by a $1 \mathrm{D}$ matrix of size $1 \times K$.

\subsection{Healthcare monitoring using backtracking search based artificial neural network (BS-ANN)}

In this section, we categorize the health status and provide the best guidance to the patient using the IoT Support Cloud Environment. The collected and collected data should be processed using the decision making method. Therefore, we use the artificial neural network (ANN) for the same classification. Here, we combine backtrack search with ANN for optimal solution computation process. Let $\left.p_{x}^{a, b}=\left[p_{a, b}(0, x), \ldots . p_{a, b}(B-1), x\right)\right]^{t}, 0 \leq x \leq n-1$, where $p_{x}^{a, b}$ denote the nonzero rudiments of the xth row in the time areapassage matrix $Q_{a, b}$ as exposed below:

$$
Q_{a, b}=\left[\begin{array}{lcccc}
p_{a, b}(0,0) & 0 & \ldots . & p_{a, b}(2,0) & p_{a, b}(1,0) \\
p_{a, b}(1,1) & p_{a, b}(0,1) & \ldots & p_{a, b}(3,1) & p_{a, b}(2,1) \\
\cdot & \cdot & & \cdot & \cdot \\
\cdot & \cdot & & . & \\
0 & 0 & \ldots \ldots & p_{a, b}(0, n-2) & 0 \\
0 & 0 & \ldots \ldots \ldots & p_{a, b}(1, n-1) & p_{a, b}(0, n-1)
\end{array}\right]
$$


Individually row of $Q_{a, b}$ has Y natural rudiments, as distinct in $p_{x}^{a, b}$. Consequently, nYparameter must be compute to analyze the wireless waterway in a time-varying atmosphere, which affects the computational difficulty. The background search algorithm motivates the bird not only with the attractive terms it can produce, but also with its violent reproductive approach. Some biological groups, such as collateral cranes, lay eggs in public cages, while others may destroy their eggs. With the background search method, the nests with the best solution will be the best nests, i.e. the nests are the solution. In this paper, the network virtualization system is integrated into the search for the optimal planning context. Let the time-domain matrix $Q_{a, b}$ is parameterized, by using a fewquantities of its rows, which is represent by $\mathrm{M}$. This puts Mmarkerbodily in the time field in which the number of needs is intended. After that, the number of needs at other times is interpolated by utilize these time-domain marker. This thought minimizes the digit of limit to be compute from nY to MYwhere $M<<n$. Hence, we first select M rows of $Q_{a, b}$, which are represent by $p_{m(1)}^{a, b}, \ldots ., p_{m(M)}^{a, b}$ as Mmarker for the time sphereindicatorin $Q_{a, b}$. After that, we describe $M_{a, b}:=[m(1), \ldots, m(M)]$ as set of this row indicator of the time-domainmarker. Every channel $p_{x}^{a, b}$, where $x \ni M_{a, b}$, can be spoken as a linear amalgamation of these $\mathrm{M}$ markers $p_{m(1)}^{a, b}, \ldots, p_{m(M)}^{a, b}:$

$$
p_{a, b}(z, x)=q_{z, x, a, b}^{t}\left[p_{a, b}(z, m(1)), \ldots \ldots \ldots, p_{a, b}(z, m(M))\right]^{t}
$$

For $0 \leq l \leq L-1$ where

$$
q_{z, x, a, b}=\left[q_{z, x, a, b}(m(1)), \ldots \ldots \ldots \ldots, q_{z, x, a, b}(m(M))\right]^{t}
$$

is an $M \times 1$ vector of time-domain exclamation weights used to state the number of needsamong ath convey antenna and $b t h$ accept antenna at time x.Fordropping the digit of interruption weights that is mandatory to design each appeal and is spoken as $p_{a, b}(z, x)$ at time $\mathrm{x}$ as an outburstamong two timedomain markers, comparable to the zth path at time $m(c)$ and $m\left(c^{\prime}\right)$, that is $p_{a, b}(z, m(c))$ and $p_{a, b}\left(z, m\left(c^{\prime}\right)\right)$, where $m(c), m\left(c^{\prime}\right) \in M_{a, b}$. The two indicators are chosen from $M_{a, b}$, in such a way that they have the utmostassociation with $p_{a, b}(z, x)$. This gives suitableoutcome for the variety of two markers in $M_{a, b}$, which are neighboring to the own channel coefficient. Hence, the digit of markers required in the exclamation process can be abridged from $\mathrm{M}$ to 2 . The shout weight vector $q_{z, x, a, b}$ in eq. (8) is simplifiedto have only two non-zero factors $q_{z, x, a, b}(m(c))$ and $q_{z, x, a, b}\left(m\left(c^{\prime}\right)\right)$. The other basics are set to be zero. Hence, believe that the friendship between $p_{a, b}(z, m)$ and $p_{a, b}(z, x)$ can be affirmed as follow:

$$
R[m, x]:=U\left[p_{a, b}(z, m) p_{a, b}^{P}(z, x)\right.
$$

After that the inference of the optimal exclamation weight is undemanding. Next, set of non-zero exclamation weight $b_{l, n, p, q}^{N E}$ that minimize the networkassessmentfault is definite as,

$$
U\left[\left|p_{a, b}(z, x)-q_{z, x, a, b}^{n u}{ }^{P} \tilde{p}_{x, a, b}(z)\right|^{2}\right]
$$


It is simplify by orthogonality attitude as follow:

$$
q_{z, x, a, b}^{n u}{ }^{J}=P_{x, p \tilde{p}}^{a, b} \underset{x, \tilde{p} \tilde{p}}{P^{a, b}-1}
$$

where $P_{x, \tilde{p}}^{a, b}=U\left[p_{a, b}(z, x) \tilde{p}_{x, a, b}^{P}(z)\right]$, and $\underset{n P_{n \tilde{p}}^{a, b}}{a \tilde{p}}=U\left[\tilde{p}_{x, a, b}(z, x) \tilde{p}_{x, a, b}^{P}(z)\right]$

Then, result can be printed as,

$$
P_{x, p \tilde{p}}^{a, b}=\left[R[m(c), x), R\left[m\left(c^{\prime}\right), x\right]\right]
$$

Finally, the resultant $\mathrm{R}$ matrices utilized to perform schedule as per the user requests. The constraints are optimized by the ANN. The originalinhabitants of $\mathrm{m}$ points arearbitrarilygenerated in the set $(\mathrm{S})$. Each point $x_{i}$ in the inhabitants is compute as follows:

$$
n_{r}^{T} \leftarrow x_{T}+\delta\left(r_{T}-x_{T}\right) ; T=1,2, \mathrm{~K}, x
$$

Where $\delta$ is presumed behomogeneouslyscatteredamong 0 and $1, r_{t}$ and $x_{t}$ denote the upper andMinimal control of the initial phase. The finest and most horrible performance principles are calculated as follow:

$$
\begin{aligned}
& F_{q}=\operatorname{MIN}\left\{F\left(n_{r}\right) ; r=1,2, \mathrm{~K}, x\right\} \\
& F_{v}=\operatorname{MAX}\left\{F\left(n_{r}\right) ; r=1,2, \mathrm{~K}, x\right\}
\end{aligned}
$$

The consistent visual significance of the entire inhabitants depends on the limitations of the difficulty defined by the fashionable.

$$
D=\underset{T \in\{1, \mathrm{~K}, x\}}{M A X}\left(r_{T}-x_{T}\right)
$$

When D is unfilled, the population point move approximately, or else you choose a point at random. Search behavior can be understood as restricted behavior. When D is not unfilled, the algorithm initiates the looking independence and chaotically chooses a summit within the visualrange, i.e., an index is casuallychosen and the summit $x_{i}$ is enthused towards it if the stipulation $F\left(n_{m}\right)<F\left(n_{r}\right)$ hold. The track of group is denoting as follows:

$$
D I R_{r}=n_{m}-n_{r}
$$

The way of group used to calculate the position of a new summit is called the test point, which is called the optimal performance. At one point, consider moving to the lowest operating $n_{M I N}$ charge point. Here's how to calculate a new test point

$$
D I R_{r}=n_{M I N}-n_{r}
$$


Elements at any particular point or in any particular direction activate search engines and provide general access to the top and bottom of the package. Whether the newly created tests $T_{r}$ are selected for the next step depends on the situation

$$
n_{r}= \begin{cases}T_{r} ; & \text { if } F\left(T_{m}\right)<F\left(n_{r}\right) \\ n_{r} ; & \text { otherwise }\end{cases}
$$

After optimize the time unreliableconstraint of each user calculates own strength $\left(\mathrm{V}_{\mathrm{s}}\right)$ as follows:

$$
D_{f}=n_{1}+n_{2}+\Lambda \quad f=k_{1}, k_{2}, k_{3} \ldots k_{x}
$$

Lastly, the optimal outcome select as follows:

$$
\text { or }=\max \left(D_{f}^{1}, D_{f}^{2}, D_{f}^{3}, \Lambda D_{f}^{x}\right)
$$

\section{Results and Discussion}

This section deals with the test system and discusses the evaluation of the performance of the specific scheme. The test section is alienated into four sections: data mining, data granulation performance, blood pressure attack prediction analysis, and warning generation statistical analysis. Ideal for cloud applications that include diverse, dynamic, and built-in (QoS) client requirements. Different applications have different operating levels, workloads, and dynamic program scale requirements; however these features create an ambiguous situation when both management models and enterprise models use the cloud for host applications. The cloud creates complex connections, sends and organizes needs. Cloud SIM is considered as a popular tool for displaying and reproducing cloud dispensationcircumstances. It provides structure and social performance of obscure discovery areas. Simulate cloud conditions and programs to evaluate the effectiveness of relevant knowledge to explore such a powerful, highly explicit and multifaceted scenario. A database of consumer symptoms and high blood pressure activity is not available in the database on the Internet. Incompatibility forced the integration of different databases to evaluate the specific structure of the database. It uses three different databases: the Diabetes Database, the Chronic Kidney Database, and the Functional Database. This data is systematically integrated into the Chronic Kidney Disease Database, Diabetes Database, and Functional Database (UCI Machine Learning Repository, (2017A, B)) (UCI Machine Learning Repository, (2017E)) to check the performance of a specific system.

- Application domain: It gives information about a particular application domain on which a study has been carried out.

- Major contribution: It provides relevant information on the contribution made in a particular research.

- IoT sensor: IoT sensor technology is used to collect datasets pertaining to health, environment, location and dietary conditions of the user at any time anywhere.

- Real time perspective: It provides timely information pertaining to current condition of the user at a given point of time.

- Prediction model: This predicts an architecturally effective behavior. Therefore, as health is a dynamic option, it is important to make effective predictions to ensure better utilization in this important program area.

- Cloud storage: It enables the storage of a large quantity of data at cloud folder which can be accessed anywhere at any time. 


\subsection{Dataset description}

After spreading to the neural network, three layers are used to predict blood pressure, namely: input, nigh and output layers, as shown in the figure. 2. There are one in nine injections to predict the severity of an attack of hypertension. Eight of the nine entry points are sickness, heart illness, liver disease; blood glucose, kidney disease, irregular heartbeat, severe headache, obesity, and the rest are hypertension. BS$\mathrm{AN}$ is isolated in the form of high risk, low risk, moderate risk or high risk of hypertension. Of the 5000 data sets, 3000 data sets are used for preparation data and the rest are used for test data. Trained at BSANN Speed 3.7 with 3000 sets of data with different motivations, learning speeds and different number of repetitions. Initially, the trial case is performed over 1000 repetitions with different repetition speeds and learning speeds. Their values are shown in Table 1. Test results show that the total square error decreases by 0.65 speeds and 0.35 learning speed. In the case of the second experiment, 2000 repetitions are used with different learning speeds and speeds. The results show that the lowest error occurred at 0.15 and 0.85 knowledge speeds. The third test results show a lower quadratic error -0.15 speeds and 0.85 knowledge speed. In the next test, the same values of speed and knowledge coefficients are used for 4000 repetitions. The results show the lowest errors with an error of 0.05 and a learning speed of 0.95 . After 4000 repetitions, 5000 and 6000 repetition tests are performed and the minimum error values are determined as 0.0 speed values 1.015 and 1.013 and 0.85 learning speed. At 5000 and 6000 repeat tests we see that there is significant change in the total rectanglemistake. Therefore, it is recommended to use 5000 repeat tests with 0.15 study rate and 0.85 notation rates. After the database was prepared, an audit was conducted to examine 2000 databases according to the risk of hypertensive attack. BS-ANN can be used to calculate the risk of hypertension in the form of various statistical measures. Properly classified health records, kappa statistics, average complete error, relation absolute mistake, root standard square error, root relation square fault, case defense.

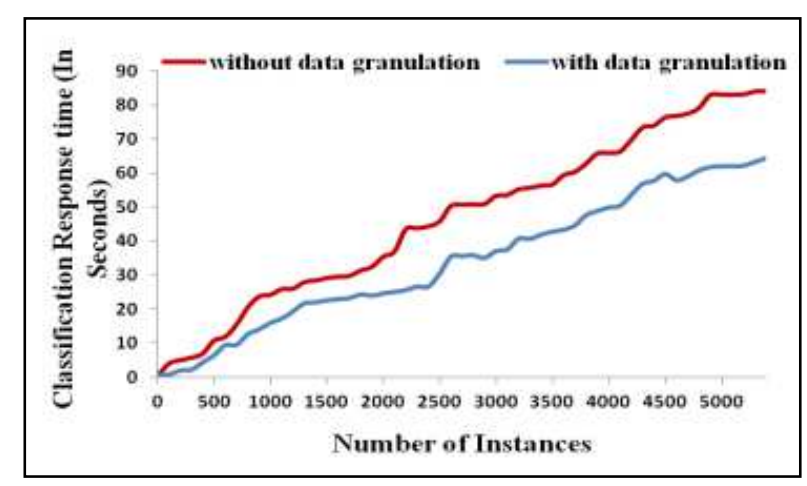

Fig. 2 Classification rejoinder time with or without data granulation

Table 1 Total square error for different iterations 


\begin{tabular}{|c|c|c|c|c|c|c|c|}
\hline \multirow[t]{4}{*}{ Momentum } & \multirow{4}{*}{$\begin{array}{l}\text { Learning } \\
\text { Rate }\end{array}$} & \multicolumn{6}{|c|}{ Total Square Error } \\
\hline & & (a) & (b) & (c) & (d) & (e) & (f) \\
\hline & & 1000 & 2000 & 3000 & 4000 & 5000 & 6000 \\
\hline & & \multicolumn{2}{|c|}{ Iterations Iterations } & Iterations & Iterations & Iterations & Iterations \\
\hline 0.05 & 0.95 & 5.861 & 4.561 & 5.012 & 3.123 & 1.915 & 1.713 \\
\hline 0.15 & 0.85 & 5.824 & 4.212 & 3.118 & 4.813 & 1.015 & 1.013 \\
\hline 0.25 & 0.75 & 5.483 & 5.701 & 4.215 & 4.541 & 2.818 & 1.890 \\
\hline 0.35 & 0.65 & 5.988 & 5.270 & 4.581 & 4.818 & 2.903 & 2.918 \\
\hline 0.45 & 0.55 & 5.921 & 5.534 & 4.845 & 4.931 & 2.818 & 3.860 \\
\hline 0.55 & 0.45 & 6.254 & 5.582 & 5.055 & 4.828 & 2.880 & 3.900 \\
\hline 0.65 & 0.35 & 5.215 & 5.287 & 5.621 & 4.917 & 3.907 & 2.807 \\
\hline 0.75 & 0.25 & 6.248 & 5.312 & \begin{tabular}{|l|}
5.331 \\
\end{tabular} & 5.218 & 4.559 & 4.570 \\
\hline 0.85 & 0.15 & 7.185 & 6.122 & 6.114 & 5.618 & 4.618 & 5.850 \\
\hline 0.95 & 0.05 & 8.213 & 7.283 & 7.517 & 6.180 & 5.188 & 5.958 \\
\hline
\end{tabular}

BS-ANN provides a high specificity of $91.20 \%$, high sensitivity of $92.80 \%$, precision of $90.6 \%$, high recall of $90.4 \%$ and high F-measure of $89.6 \%$ than other classification models i.e. deep neural network (DNN) [34], multilayer perceptron (MLP) [35], K-nearest neighbor (KNN) [36]and linear regression (LR) [37]. Get useful features to determine the level and risk of hypertension in a healthy fog system. This type of performance is calculated based on the total time of the two factors, taking into account other aspects of health and work. at the start, the system was in progress with 500 requests, and then after 20 minutes the system was tested on 500 requests and the efficiency of the system was increased to 200 minutes. The accuracy of categorization models shows BS-ANN performs better than all other models.

\subsection{Performance comparison}

The system analytically analyzes the performance of the warning generation. The specific model learns differentnumericalparameter such as compassion, uniqueness, accuracy, security, average completemistake, root typical square mistake, root comparative quadratic mistake, and comparativecomplete error. Table 3 illustrates the statistical results obtained for the specific organization throughout the monitoring training. The elevatedprice of sensitivity $(89.21 \%)$, specificity (93.24\%), coverage $(95.21 \%)$ and accuracy $(92.10 \%)$ show the high efficiency of the particular model. Additionally, mistake rates are lower in the process of generating warnings in the form of average completemistake, root standardtetragonmistake, root square mistake, and completemistake.

Table 2 Performance comparison of proposed and existing classifiers

\begin{tabular}{lllllll}
\hline Metrics (\%) & ANN & KNN & MLP & LR & DNN & BS-ANN \\
\hline Accuracy & 90.12 & 91.52 & 92.1 & 92.5 & 93.4 & 93.49 \\
Precession & 89.25 & 92.3 & 92.5 & 91.9 & 92.9 & 94.19 \\
Sensitivity & 89.21 & 89.29 & 89.34 & 89.72 & 91.21 & 93.25 \\
Specificity & 93.21 & 93.24 & 94.39 & 95.02 & 95.35 & 95.69 \\
Mean absolute error & 79.05 & 79.95 & 79.98 & 81.05 & 82.15 & 83.52 \\
Recall & 89.12 & 89.25 & 89.39 & 80.98 & 92.15 & 96.39 \\
F-measure & 94.39 & 95.89 & 96.09 & 96.31 & 96.51 & 97.31 \\
\hline
\end{tabular}

Figure 3 shows an accurate comparison of specific and current taxonomies. The plot states that the specific PS-ANN accuracy is very high compared to the current complex classifiers. Figure 4 shows a previous comparison of specific and current taxonomies. The plot makes it clear that the proposed BSANN predecessor surpasses the current state-of-the-art classifiers. Figure 5 shows a comparison of the sensitivity of specific and existing classifiers. The plot makes it clear that the specific BS-ANN sensitivity is very elevatedcompare to obtainable complex classfires. Figure 6 shows a specific comparison of specific and current taxonomies. The plot makes it clear that the specificity of the planned BS-ANN is very high compare to the obtainable complex classifiers. Figure 7 shows a complete false comparison of 
specific and existing classifiers. The specific PS-ANN average absolute error plot clearly reflects when compared to existing complex classifiers. Figure 8 shows a comparison of specific and current taxonomies. The plot makes it clear that the proposed BS-ANN recall is much higher than the current complex classfire. Figure 9 shows a comparison of the f-dimensions of specific and existing classfires. The plot shows that the scale of the proposed BS-AN is much higher than the current modern class fires.

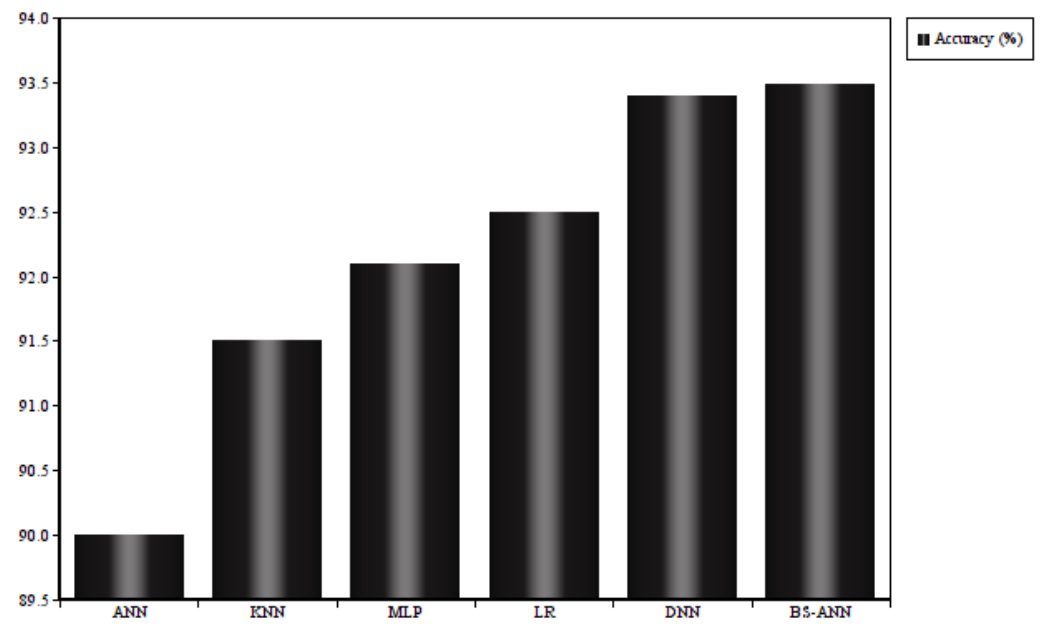

Fig. 3 Accuracy comparison of proposed and existing classifiers

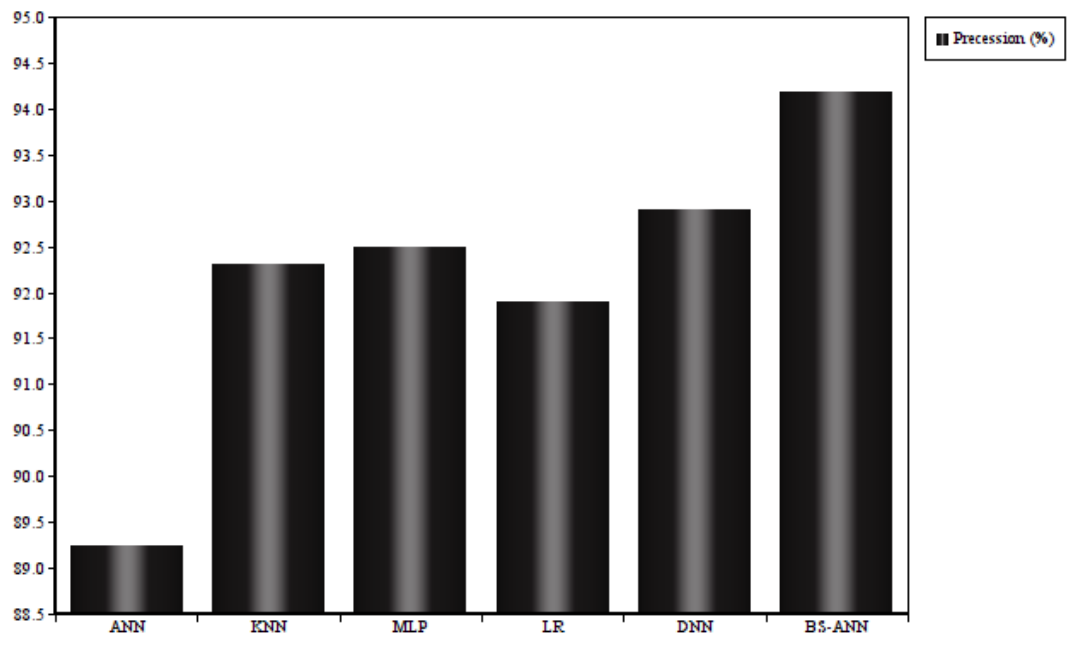

Fig. 4 Precession comparison of proposed and existing classifiers 


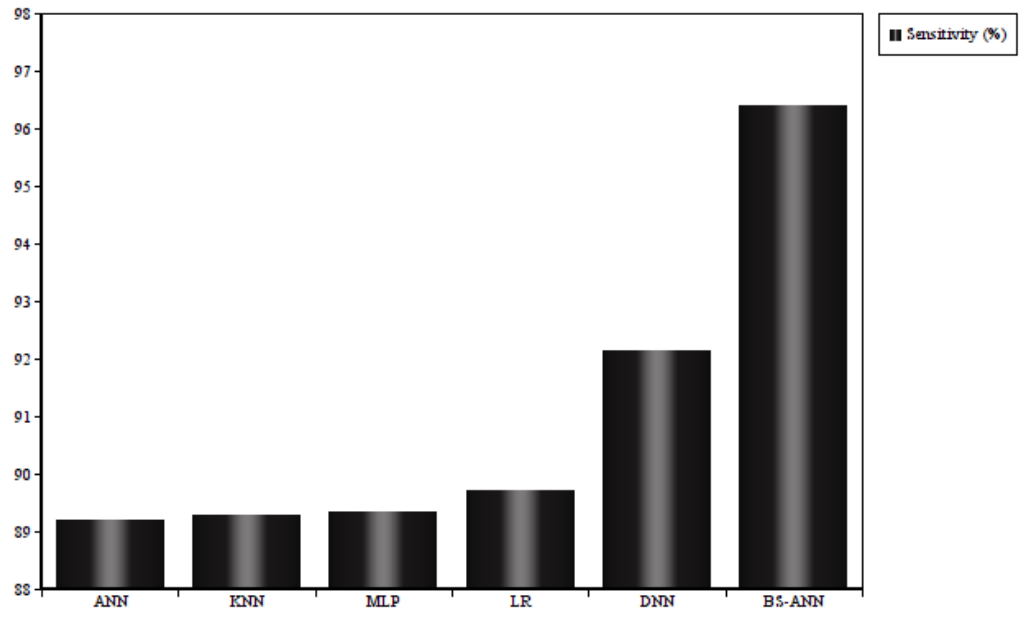

Fig. 5 Sensitivity comparison of proposed and existing classifiers

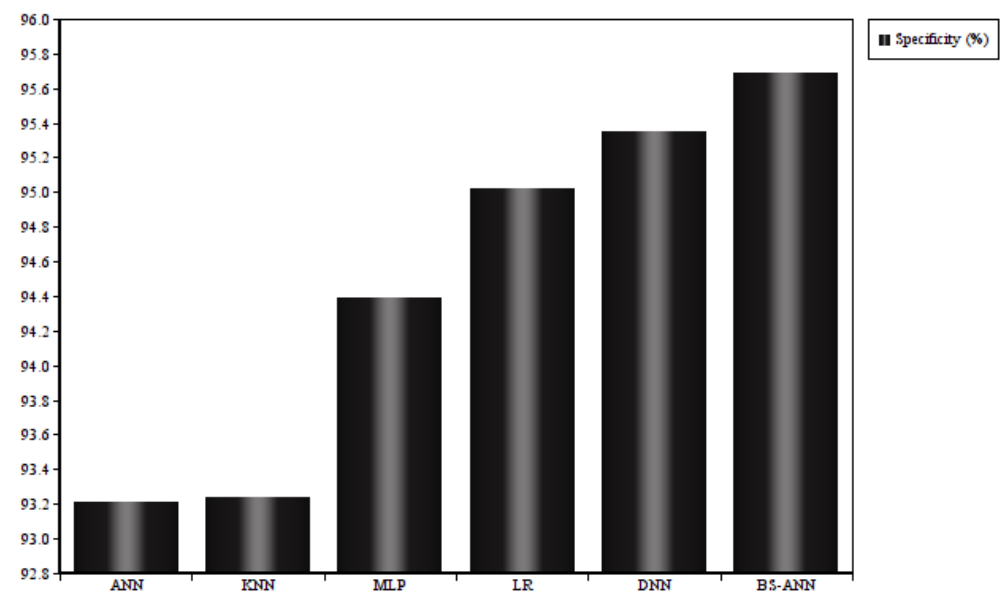

Fig. 6 Specificity comparison of proposed and existing classifiers

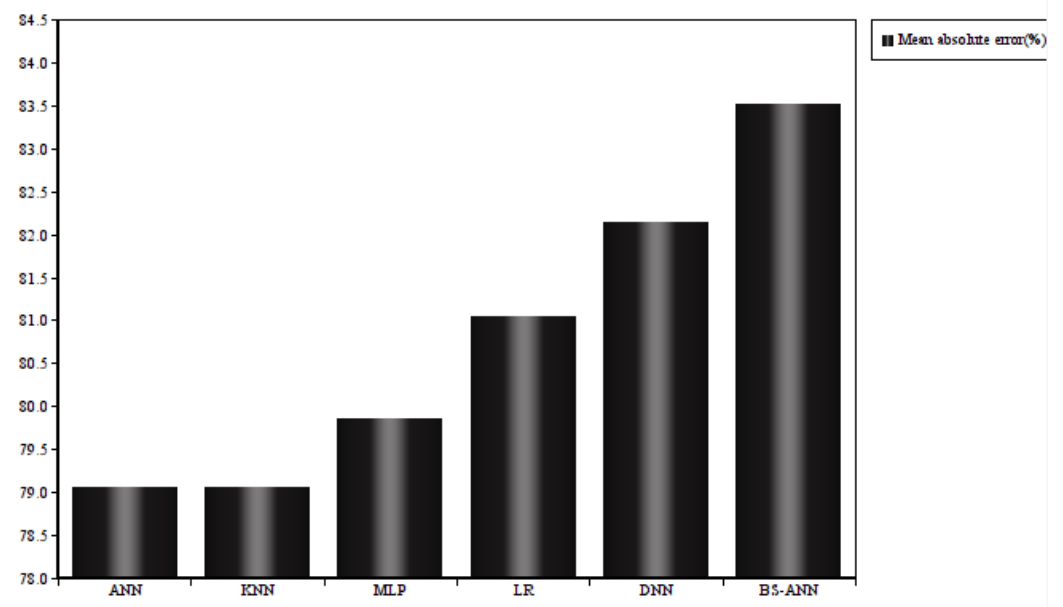

Fig. 7 Mean absolute error comparison of proposed and existing classifiers 


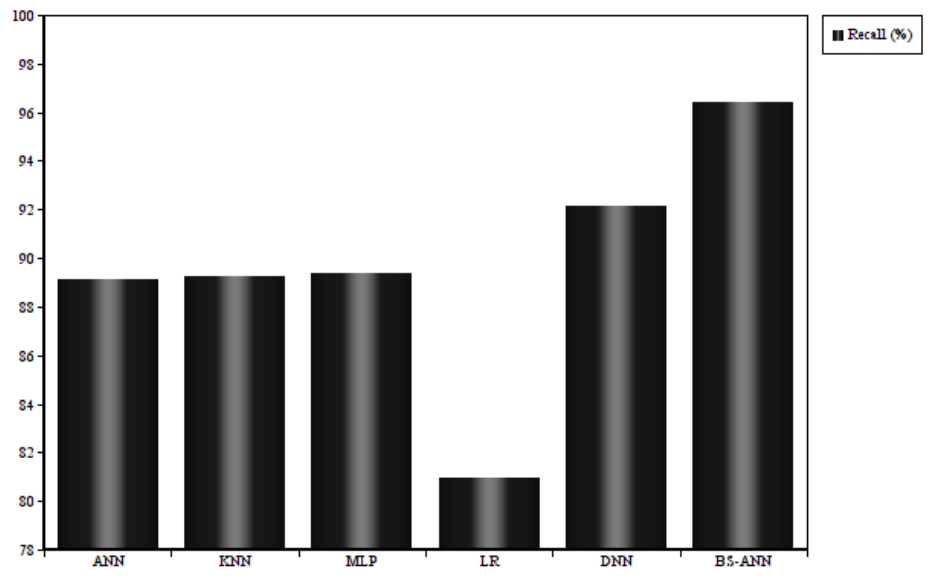

Fig. 8 Recall comparison of proposed and existing classifiers

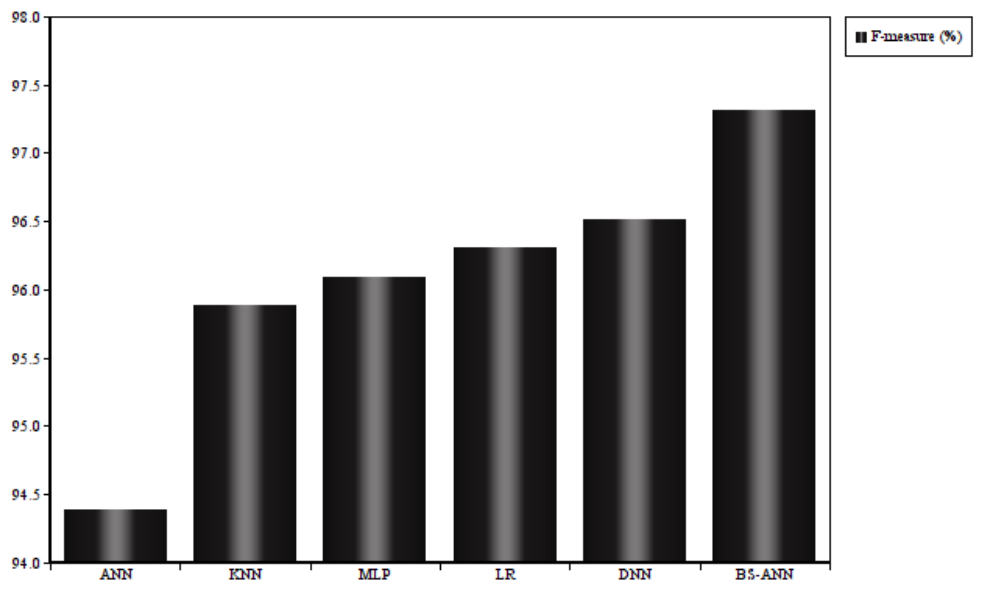

Fig. 9 F-measure comparison of proposed and existing classifiers

\section{Conclusion}

Iotbased health monitoring systems are important research because they are used in cloud computing, which can improve data size when using cloud data storage. This study focuses on effective data science technology for IoT-based health monitorsystem, which will increase the efficiency and efficiency of data processing. Sensors are located on the human body to gather all the medical data of the enduring and store this information in the cloud. First, we developed an advanced PAV optimization algorithm for cloud data clustering that can improve forecast speeds. We have demonstrated the optimal extraction and sampling techniques for obtaining features from the system and selecting the required characteristics. We use the BS-DNN human health classification. We got better results compared to other health systems. 


\section{Funding}

This study was not funded by any organization.

\section{Authors' Contributions}

Both the authors contributed equally to bring this manuscript in its final version from idea generation, conducting literature review, designing as well executing the proposed system and drafting the article with the derived result set.

\section{Conflicts of Interest}

The authors declare that there is no conflict of interests regarding the publication of this paper.

\section{Data Availability \& Code Availability}

The data that support the findings of this study are available from the corresponding author upon reasonable request.

\section{References}

1. Catarinucci, L., De Donno, D., Mainetti, L., Palano, L., Patrono, L., Stefanizzi, M.L. and Tarricone, L., 2015. An IoT-aware architecture for smart healthcare systems. IEEE internet of things journal, 2(6), pp.515-526.

2. Amendola, S., Lodato, R., Manzari, S., Occhiuzzi, C. and Marrocco, G., 2014. RFID technology for IoT-based personal healthcare in smart spaces. IEEE Internet of things journal, 1(2), pp.144152.

3. He, D. and Zeadally, S., 2014. An analysis of RFID authentication schemes for internet of things in healthcare environment using elliptic curve cryptography. IEEE internet of things journal, 2(1), pp.72-83.

4. Yao, Q., Han, X., Ma, X.K., Xue, Y.F., Chen, Y.J. and Li, J.S., 2014. Cloud-based hospital information system as a service for grassroots healthcare institutions. Journal of medical systems, 38(9), p.104.

5. Mu-Hsing, K., 2012. A healthcare cloud computing strategic planning model. In Computer Science and Convergence (pp. 769-775). Springer, Dordrecht.

6. Hanner, N., Ermakova, T., Repschlaeger, J. and Zarnekow, R., 2014. Designing a Business Model for a Cloud Marketplace for Healthcare. In Trusted Cloud Computing (pp. 285-294). Springer, Cham.

7. Xu, X. and Zhong, M., 2014. Wireless body sensor networks with cloud computing capability for pervasive healthcare: Research directions and possible solutions. In Frontier and Future Development of Information Technology in Medicine and Education (pp. 979-988). Springer, Dordrecht.

8. Poulymenopoulou, M., Malamateniou, F. and Vassilacopoulos, G., 2012. Emergency healthcare process automation using mobile computing and cloud services. Journal of medical systems, 36(5), pp.3233-3241.

9. Cagalaban, G., Kim, D., Kim, S. and Choi, E.J., 2012. A Multilevel Security Framework for Cloud-Based Ubiquitous Healthcare Application Service. In Computer Applications for Security, Control and System Engineering (pp. 168-175). Springer, Berlin, Heidelberg.

10. Severi, S., Sottile, F., Abreu, G., Pastrone, C., Spirito, M. and Berens, F., 2014, June. M2M technologies: Enablers for a pervasive Internet of Things. In 2014 European Conference on Networks and Communications (EuCNC) (pp. 1-5). IEEE. 
11. Zdravković, M., Noran, O. and Trajanović, M., 2014. Interoperability as a property of ubiquitous healthcare systems. IFAC Proceedings Volumes, 47(3), pp.7849-7854.

12. Vega, D.E., 2012. Automated interoperability testing of healthcare information systems. In Advances in Computers (Vol. 85, pp. 213-276). Elsevier.

13. Saha, R., Kumar, G., Rai, M.K., Thomas, R. and Lim, S.J., 2019. Privacy Ensured \$\{e\} \$Healthcare for Fog-Enhanced IoT Based Applications. IEEE Access, 7, pp.44536-44543.

14. Alharbi, F., Atkins, A. and Stanier, C., 2017. Cloud computing adoption in healthcare organisations: a qualitative study in Saudi Arabia. In Transactions on Large-Scale Data-and Knowledge-Centered Systems XXXV (pp. 96-131). Springer, Berlin, Heidelberg.

15. Wang, X. and Cai, S., 2020. Secure healthcare monitoring framework integrating NDN-based IoT with edge cloud. Future Generation Computer Systems.

16. Abdelmoneem, R.M., Benslimane, A. and Shaaban, E., 2020. Mobility-Aware Task Scheduling in Cloud-Fog IoT-Based Healthcare Architectures. Computer Networks, p.107348.

17. Darwish, A., Hassanien, A.E., Elhoseny, M., Sangaiah, A.K. and Muhammad, K., 2019. The impact of the hybrid platform of internet of things and cloud computing on healthcare systems: opportunities, challenges, and open problems. Journal of Ambient Intelligence and Humanized Computing, 10(10), pp.4151-4166.

18. Kumar, P.M., Lokesh, S., Varatharajan, R., Babu, G.C. and Parthasarathy, P., 2018. Cloud and IoT based disease prediction and diagnosis system for healthcare using Fuzzy neural classifier. Future Generation Computer Systems, 86, pp.527-534.

19. Verma, P. and Sood, S.K., 2018. Cloud-centric IoT based disease diagnosis healthcare framework. Journal of Parallel and Distributed Computing, 116, pp.27-38.

20. Chaudhary, A., Choudhary, C., Gupta, M.K., Lal, C. and Badal, T., Microservices in Big Data Analytics.

21. Shastri, A.S., Thorat, E.V., Kulkarni, A.J. and Jadhav, P.S., 2019. Optimization of constrained engineering design problems using cohort intelligence method. In Proceedings of the 2nd International Conference on Data Engineering and Communication Technology (pp. 1-11). Springer, Singapore.

22. Raykar, S.S. and Shet, V.N., 2020. Design of healthcare system using IoT enabled application. Materials Today: Proceedings, 23, pp.62-67.

23. Saha, R., Kumar, G., Rai, M.K., Thomas, R. and Lim, S.J., 2019. Privacy Ensured \$ \{\} \$Healthcare for Fog-Enhanced IoT Based Applications. IEEE Access, 7, pp.44536-44543.

24. Haghi, M., Neubert, S., Geissler, A., Fleischer, H., Stoll, N., Stoll, R. and Thurow, K., 2020. A Flexible and Pervasive IoT Based Healthcare Platform for Physiological and Environmental Parameters Monitoring. IEEE Internet of Things Journal.

25. Wu, T., Wu, F., Qiu, C., Redoute, J.M. and Yuce, M.R., 2020. A Rigid-Flex Wearable Health Monitoring Sensor Patch for IoT-Connected Healthcare Applications. IEEE Internet of Things Journal.

26. Kumar, A., Krishnamurthi, R., Nayyar, A., Sharma, K., Grover, V. and Hossain, E., 2020. A Novel Smart Healthcare Design, Simulation, and Implementation Using Healthcare 4.0 Processes. IEEE Access, 8, pp.118433-118471.

27. Wang, X. and Cai, S., 2020. Secure healthcare monitoring framework integrating NDN-based IoT with edge cloud. Future Generation Computer Systems.

28. Abdelmoneem, R.M., Benslimane, A. and Shaaban, E., 2020. Mobility-Aware Task Scheduling in Cloud-Fog IoT-Based Healthcare Architectures. Computer Networks, p.107348.

29. Mutlag, A.A., AbdGhani, M.K., Arunkumar, N.A., Mohammed, M.A. and Mohd, O., 2019. Enabling technologies for fog computing in healthcare IoT systems. Future Generation Computer Systems, 90, pp.62-78.

30. Mahbub, M., Hossain, M.M. and Gazi, M.S.A., 2020. IoT-Cognizant cloud-assisted energy efficient embedded system for indoor intelligent lighting, air quality monitoring, and ventilation. Internet of Things, 11, p.100266. 
31. M. Anuradha et al., "IoT enabled Cancer Prediction System to Enhance the Authentication and Security using Cloud Computing", Microprocessors and Microsystems, p. 103301, 2020. Available: 10.1016/j.micpro.2020.103301.

32. Jiang, F., He, J. and Tian, T., 2019. A clustering-based ensemble approach with improved pigeoninspired optimization and extreme learning machine for air quality prediction. Applied Soft Computing, 85, p.105827.

33. Anh, N.X., Nataraja, R.M. and Chauhan, S., 2020. Towards near real-time assessment of surgical skills: A comparison of feature extraction techniques. Computer Methods and Programs in Biomedicine, 187, p.105234.

34. M. Anuradha et al., "IoT enabled Cancer Prediction System to Enhance the Authentication and Security using Cloud Computing", Microprocessors and Microsystems, p. 103301, 2020. Available: 10.1016/j.micpro.2020.103301.

35. S. Ravikumar, S. Chandrasekaran, S. Ramesh (2016), "Safety Assessment of Distributed Automotive Software System Model with Design for Traceability," Asian Journal of Information Technology,15(11): pp.1799-1815,2016. DOI: 10.36478/ajit.2016.1799.1815

36. A. Samydurai and Vasuhi "An Enhanced Fault-Tolerance Based E-Content Delivery System Using Range Queries on Peer-to-Peer Networks", 2016. Asian Journal of Information Technology, 15: 211-216

37. Revathi K , A.Samydurai, "Adaptive Deep Convolutional Neural network based Secure Integration of Fog to Cloud Supported IOT for Health Monitoring System", Transactions on Emerging Telecommunications Technologies, Online ISSN:2161-3915, Vol.31, Issue 10, 2020. 